\title{
TENSOR PRODUCTS OF LOCALLY CONVEX ALGEBRAS
}

\author{
HARVEY A. SMITH
}

1. Introduction. The terms locally convex algebra, locally $m$-convex algebra, and $Q$-algebra are defined in [5]. Various cross-norms on the tensor product of Banach spaces are defined in [6]. The inductive and projective tensor products of locally convex spaces are defined in [4].

Gelbaum [1], [2] and Tomiyama [8] have remarked that the tensor product of two Banach algebras completed with respect to the greatest cross-norm is again a Banach algebra. When a Banach algebra is obtained by completing the tensor product of two commutative Banach algebras with respect to a cross-norm, these authors have given sufficient conditions under which its space of nonzero multiplicative functionals with relative weak* topology can be characterized as the topological direct product of the corresponding spaces associated with the two factors. Gil de Lamadrid [3] has announced the extension of this result to the noncommutative case. In [7], various necessary and sufficient conditions are given for the characterization of Gelbaum and Tomiyama to hold.

Lemmas 1 and 2 of this paper show that if a locally convex algebra is the completion, in a topology not stronger than the inductive topology, of the tensor product of two locally convex algebras, then there is a canonical continuous one-to-one mapping of its space of nonzero continuous multiplicative linear functionals, with the weak topology induced by the algebra, into the topological direct product of the corresponding spaces associated with the two factors. If this mapping is open, it provides a characterization of the space of nonzero continuous multiplicative functionals associated with the completed tensor product algebra. Theorem 1 shows that if the completed tensor product is a locally $m$-convex $Q$-algebra, then the mapping is open. Since every Banach algebra is a locally $m$-convex $Q$-algebra, this theorem generalizes the discussion of such characterizations given in $[1],[3],[7],[8]$.

Theorem 2 shows the complete projective tensor product of locally $m$-convex algebras to be locally $m$-convex, while Theorem 3 states that if the two algebras are also complete commutative $Q$-algebras then the complete projective tensor product inherits these properties. Since the complete projective tensor product of locally convex spaces corresponds to the completion of the tensor product of Banach spaces

Received by the editors January 29, 1965. 
with the greatest cross-norm, Theorems 2 and 3 are analogous to the result that such a product of Banach algebras is again one.

Theorem 4 gives a necessary and sufficient condition for semisimplicity of the complete projective tensor product of semisimple complete commutative locally $m$-convex $Q$-algebras. It generalizes a result for Banach algebras given in [7].

It is of interest to note that the arguments used to obtain Theorems 1 and 3 are quite different from those employed to obtain the corresponding results for Banach algebras. In particular the assumption of commutativity plays an essential role in the proof of Theorem 3, and the problem of extending the theorem to the noncommutative case remains open, while the analogous result for Banach algebras does not depend on commutativity. Theorem 3 provides a method for constructing new complete commutative locally $m$-convex $Q$-algebras from old ones. An example is given at the end of the paper.

2. Notation and remarks. We follow the notation and terminology of [5] for spectrum, $\Sigma_{A}(x)$, and spectral radius, $\sigma_{A}(x)$, of an element $x$ in the algebra $A$. If $A$ is a locally convex space, $A^{\prime}$ is the dual with the weak $A$ topology. If $A$ is also an algebra, $M(A)$ is the set of nonzero multiplicative functionals in $A^{\prime}$ with the relative topology.

REMARK 1. A locally convex algebra $A$ is a $Q$-algebra iff $\sigma_{A}(x)$ is continuous at zero.

Proof. Elementary from Proposition 13.5 of [5].

REMARK 2. If $A$ is a complete locally $m$-convex algebra, then

$$
\sigma_{A}(x) \geqq \sup \{|f(x)|: f \in M(A)\}
$$

Proof. If $A$ is commutative the equality holds (Corollary 5.6 of [5]). Every commutative subalgebra of a complete locally $m$-convex algebra $A$ is contained in a maximal commutative subalgebra $B$, which is closed and hence complete. If $x \in B$, then obviously $\Sigma_{A}(x)$ $=\Sigma_{B}(x)$ and hence $\sigma_{A}(x)=\sigma_{B}(x)$. But $M(A) \subset M(B)$ in the sense that the restriction of an element of $M(A)$ is in $M(B)$; hence the inequality.

Remark 3. A complete commutative locally $m$-convex algebra, $A$, is semisimple iff $A^{\prime}$ is the closed linear hull of $M(A)$.

Proof. $A$ is semisimple iff $m(a)=0$ for all $m \in M(A)$ implies $a=0$ (Corollary 5.5 of [5]). Suppose $A^{\prime}$ is the closed linear hull of $M(A)$. For any nonzero $a \in A$ there is $f$ in $A^{\prime}$ such that $f(a) \neq 0$. For all $\epsilon>0$, there is a linear combination $g$ of elements of $M(A)$ such that $|g(a)-f(a)|<\epsilon$ so not all members of $M(A)$ can vanish at $a$. Hence $A$ is semi-simple. Conversely if $A$ is semisimple the closed linear hull of $M(A)$ is the orthogonal complement in $A^{\prime}$ of some closed subspace 
of $A$. The closed subspace of $A$ is zero by the cited corollary, so its orthogonal complement is $A^{\prime}$.

\section{Tensor products of locally convex algebras.}

Lemma 1. Let $A_{3}$ be a locally convex algebra which is the completion of the tensor product, $A_{1} \otimes A_{2}$, of two locally convex algebras in a topology not stronger than the inductive topology. Then each $m_{3} \in M\left(A_{3}\right)$ is a continuous extension of $m_{1} \otimes m_{2}$ where $m_{1} \in M\left(A_{1}\right)$ and $m_{2} \in M\left(A_{2}\right)$. Conversely, every such $m_{1} \otimes m_{2}$ which has a continuous extension to $A_{3}$ is in $M\left(A_{3}\right)$.

Proof. The last assertion is immediate, for $m_{1} \otimes m_{2}$ is multiplicative on $A_{1} \otimes A_{2}$ and since multiplication is (separately) continuous in the completion, $A_{3}$, a continuous extension must also be multiplicative. Suppose $m_{3}$ is a continuous nonzero multiplicative functional on $A_{3}$. Since $m_{3}$ cannot vanish identically on $A_{1} \otimes A_{2}$, there is $a_{1} \otimes a_{2}$ with $m_{3}\left(a_{1} \otimes a_{2}\right)$ not zero, and $m_{3}\left(a_{1}^{3} \otimes a_{2}^{3}\right)=\left[m_{3}\left(a_{1} \otimes a_{2}\right)\right]^{3} \neq 0$. Letting $m_{i}$ be defined by

$$
\begin{aligned}
& m_{1}\left(b_{1}\right)=m_{3}\left(b_{1} a_{1}^{3} \otimes a_{2}^{3}\right) / m_{3}\left(a_{1}^{3} \otimes a_{2}^{3}\right), \\
& m_{2}\left(b_{2}\right)=m_{3}\left(a_{1}^{3} \otimes b_{2} a_{2}^{3}\right) / m_{3}\left(a_{1}^{3} \otimes a_{2}^{3}\right)
\end{aligned}
$$

where $a_{i}, b_{i}, c_{i} \in A_{i}, i=1,2$; we have

$$
\begin{aligned}
m_{1}\left(b_{1} c_{1}\right) & =m_{3}\left(b_{1} c_{1} a_{1}^{3} \otimes a_{2}^{3}\right) / m_{3}\left(a_{1}^{3} \otimes a_{2}^{3}\right) \\
& =\frac{m_{3}\left(b_{1} \otimes a_{2}\right) m_{3}\left(a_{1}^{3} \otimes a_{2}\right) m_{3}\left(c_{1} \otimes a_{2}\right) m_{3}\left(a_{1}^{3} \otimes a_{2}^{3}\right)}{m_{3}\left(a_{1}^{3} \otimes a_{2}^{3}\right) m_{3}\left(a_{1}^{3} \otimes a_{2}^{3}\right)} \\
& =\frac{m_{3}\left(b_{1} a_{1}^{3} \otimes a_{2}^{3}\right) m_{3}\left(c_{1} a_{1}^{3} \otimes a_{2}^{3}\right)}{m_{3}\left(a_{1}^{3} \otimes a_{2}^{3}\right) m_{3}\left(a_{1}^{3} \otimes a_{2}^{3}\right)} \\
& =m_{1}\left(b_{1}\right) m_{1}\left(c_{1}\right) .
\end{aligned}
$$

By a similar calculation $m_{2}$ is multiplicative. Suppose $m_{3}\left(d_{1} \otimes d_{2}\right) \neq 0$. Then an easy computation, using similar manipulations, yields

$$
m_{3}\left(b_{1} a_{1}^{3} \otimes a_{2}^{3}\right) / m_{3}\left(a_{1}^{3} \otimes a_{2}^{3}\right)=m_{3}\left(b_{1} d_{1}^{3} \otimes d_{2}^{3}\right) / m_{3}\left(d_{1}^{3} \otimes d_{2}^{3}\right)
$$

so $m_{1}$ is well defined. A corresponding identity shows $m_{2}$ to be well defined also. Now, by definition, $m_{1} \otimes m_{2}\left(b_{1} \otimes b_{2}\right)=m_{1}\left(b_{1}\right) m_{2}\left(b_{2}\right)$. By the preceding remark we can choose $a_{1}$ in the definition of $m_{1}$ and $m_{2}$ to be $b_{1}$ unless $m_{3}\left(b_{1} \otimes a_{2}\right)=0$ for all $a_{2} \in A_{2}$. But in that case, 


$$
m_{1}\left(b_{1}\right)=m_{3}\left(b_{1} \otimes a_{2}\right) m_{3}\left(a_{1}^{3} \otimes a_{2}^{2}\right) / m_{3}\left(a_{1}^{3} \otimes a_{2}^{3}\right)=0
$$

so

$$
m_{3}\left(b_{1} \otimes b_{2}\right)=0=m_{1}\left(b_{1}\right) m_{2}\left(b_{2}\right)=m_{1} \otimes m_{2}\left(b_{1} \otimes b_{2}\right) .
$$

Otherwise, choosing $a_{1}=b_{1}$, we get

$$
m_{1} \otimes m_{2}\left(b_{1} \otimes b_{2}\right)=\frac{m_{3}\left(b_{1}^{4} \otimes a_{2}^{3}\right)}{m_{3}\left(b_{1}^{3} \otimes a_{2}^{3}\right)} \frac{m_{3}\left(b_{1}^{3} \otimes b_{2} a_{2}^{3}\right)}{m_{3}\left(b_{1}^{3} \otimes a_{2}^{3}\right)}=m_{3}\left(b_{1} \otimes b_{2}\right) .
$$

By linearity and continuity of $m_{3}$ we have $m_{3}$ a continuous extension of $m_{1} \otimes m_{2}$. By hypothesis, the topology of $A_{3}$ is not stronger than the inductive topology on $A_{1} \otimes A_{2}$. By definition of the inductive topology, $m_{3}\left(b_{1} \otimes b_{2}\right)$ is separately continuous in $b_{1}$ and $b_{2}$, which is to say that the $m_{1}$ and $m_{2}$ defined above are continuous on $A_{1}$ and $A_{2}$.

LEMMa 2. Under the assumptions of Lemma 1 the mappings from $M\left(A_{3}\right)$ to $M\left(A_{1}\right)$ and $M\left(A_{2}\right)$ defined in the proof of Lemma 1, are continuous.

Proof. We prove only that the mapping from $M\left(A_{3}\right)$ to $M\left(A_{1}\right)$ is continuous. In the proof of Lemma $1, m_{1}$ was defined as a function of $m_{3}$ by an equation equivalent to

$$
m_{1}\left(b_{1}\right)=m_{3}\left(b_{1} \otimes a_{2}\right) m_{3}\left(a_{1}^{3} \otimes a_{2}^{2}\right) / m_{3}\left(a_{1}^{3} \otimes a_{2}^{3}\right) .
$$

This can be written dually as

$$
b_{1}\left(m_{1}\right)=\left[b_{1} \otimes a_{2}\left(m_{3}\right)\right]\left[a_{1}^{3} \otimes a_{2}^{2}\left(m_{3}\right)\right] /\left[a_{1}^{3} \otimes a_{2}^{3}\left(m_{3}\right)\right] .
$$

By definition of the weak topology, elementary theorems on continuity of combinations of complex valued functions and the fact that for each $m_{3}$ we can choose $a_{1} \otimes a_{2}$ so that, on a neighborhood of $m_{3}, m_{3}\left(a_{1} \otimes a_{2}\right)$ is bounded from zero, we have the composite function $b_{1}\left(m_{1}\left(m_{3}\right)\right)$ continuous in $m_{3}$ for each $b_{1}$ in $A_{1}$. The topology of $M\left(A_{1}\right)$ is precisely the weak topology induced by the family of functions $b_{1}\left(m_{1}\right)$. The inverse images of open sets of complex numbers under these functions form a subbase for this topology. By continuity of the composite function, the inverse images of this subbase in $M\left(A_{3}\right)$ must be open. Thus the mapping from $M\left(A_{3}\right)$ to $M\left(A_{1}\right)$ is continuous.

Theorem 1. Let $A_{3}$ be a locally m-convex $Q$-algebra which is the completion, in a topology not stronger than the inductive topology, of the tensor product $A_{1} \otimes A_{2}$ of two locally convex algebras. Then $M\left(A_{3}\right)$ is canonically homeomorphic to the subset of $M\left(A_{1}\right) \times M\left(A_{2}\right)$ consisting of elements $\left(m_{1}, m_{2}\right)$ such that $m_{1} \otimes m_{2}$ has a continuous extension to $A_{3}$. 
Proof. Lemmas 1 and 2 give the existence of a canonical one-to-one continuous map of $M\left(A_{3}\right)$ onto the designated subset of $M\left(A_{1}\right)$ $\times M\left(A_{2}\right)$ with the relative product topology, so we need only show the continuity of the inverse. This inverse mapping carries $\left(m_{1}, m_{2}\right)$ to $m_{3}$, the continuous extension of $m_{1} \otimes m_{2}$. Every neighborhood of $m_{3}$ in $M\left(A_{3}\right)$ contains an open set of the form

$$
V=\left\{m_{3}^{\prime}:\left|m_{3}^{\prime}\left(x_{j}\right)-m_{3}\left(x_{j}\right)\right|<\epsilon, \quad j=1,2, \cdots, k\right\}
$$

where $x_{j}$ are members of $A_{3}$. Since $A_{3}$ is a $Q$-algebra, by Remark 1 we have $\sigma_{A_{z}}(x)$ continuous at zero, so the set

$$
U_{j}=\left\{y: \sigma_{A_{2}}\left(x_{j}-y\right)<\epsilon / 4\right\}
$$

is a neighborhood of $x_{j}$. Since $A_{1} \otimes A_{2}$ is dense in $A_{3}$, there is $y_{j} \in A_{1} \otimes A_{2}$ in $U_{j}$ such that $m_{3}\left(y_{j}\right) \neq 0$. By Remark 2 and the fact that $A_{3}$ is complete and locally $m$-convex, we have

$$
\left|m_{3}^{\prime}\left(x_{j}\right)-m_{3}^{\prime}\left(y_{j}\right)\right|=\left|m_{3}^{\prime}\left(x_{j}-y_{j}\right)\right| \leqq \sigma_{A_{3}}\left(x_{j}-y_{j}\right)<\epsilon / 4
$$

for all $m_{3}^{\prime}$ in $M\left(A_{3}\right)$.

Let

Then

$$
y_{j}=\sum_{i=1}^{n_{j}} a_{i j} \otimes b_{i j}
$$

$$
\begin{aligned}
& \left|m_{3}^{\prime}\left(y_{j}\right)-m_{3}\left(y_{j}\right)\right| \\
& \quad=\left|\sum_{i=1}^{n_{j}} m_{1}^{\prime}\left(a_{i j}\right)\left(m_{2}^{\prime}\left(b_{i j}\right)-m_{2}\left(b_{i j}\right)\right)+\left(m_{1}^{\prime}\left(a_{i j}\right)-m_{1}\left(a_{i j}\right)\right) m_{2}\left(b_{i j}\right)\right| .
\end{aligned}
$$

Let $M=\max _{i, j}\left\{\left|m_{1}\left(a_{i j}\right)\right|,\left|m_{2}\left(b_{i j}\right)\right|\right\}, n=\max _{j}\left\{n_{j}\right\}$. Then if

$$
\left|m_{1}^{\prime}\left(a_{i j}\right)-m_{1}\left(a_{i j}\right)\right|<\min \left\{\frac{\epsilon}{4 n M}, M\right\}
$$

and

$$
\left|m_{2}^{\prime}\left(b_{i j}\right)-m_{2}\left(b_{i j}\right)\right|<\frac{\epsilon}{8 n M},
$$

we have

$$
\left|m_{3}^{\prime}\left(y_{j}\right)-m_{3}\left(y_{j}\right)\right|<\frac{\epsilon}{2} .
$$

Using the inequality $\left(^{*}\right)$ satisfied in choosing $y_{j}$, we conclude that $\left|m_{3}^{\prime}\left(x_{j}\right)-m_{3}\left(x_{j}\right)\right|<\epsilon$. Now let $\left(m_{1}^{\prime}, m_{2}^{\prime}\right)$ be in the neighborhood of 
$\left(m_{1}, m_{2}\right)$ defined in the prescribed subset of $M\left(A_{1}\right) \times M\left(A_{2}\right)$ by

and

$$
\left|m_{1}^{\prime}\left(a_{i j}\right)-m_{1}\left(a_{i j}\right)\right|<\min \left\{\frac{\epsilon}{4 n M}, M\right\}
$$

$$
\left|m_{2}^{\prime}\left(b_{i j}\right)-m_{2}\left(b_{i j}\right)\right|<\frac{\epsilon}{8 n M}
$$

for $i=1,2, \cdots, n_{j} ; j=1,2, \cdots, k$. By assumption, $m_{1}^{\prime} \otimes m_{2}^{\prime}$ has a continuous extension $m_{3}^{\prime}$ and $m_{1} \otimes m_{2}$ has the continuous extension $m_{3}$. The preceding calculation shows that $m_{3}^{\prime}$ lies in the open set $V$. The continuity of the inverse mapping has thus been shown.

4. Tensor products of locally $m$-convex algebras.

THEOREM 2. The (complete) projective tensor product of two locally $m$-convex algebras is again locally $m$-convex.

Proof. An algebra is locally $m$-convex iff its topology is defined by a family of seminorms which are submultiplicative, i.e. which satisfy the multiplicative inequality for Banach algebra norms. Choosing such a family in each factor, repetition of the argument of Lemma 2 in [9] shows that the corresponding family of seminorms generating the topology of the projective tensor product (constructed in Proposition 2 of [4]) will also be submultiplicative. The completion is clearly also locally $m$-convex.

CoRollary 1. If $A_{3}$ is the complete projective tensor product of two locally m-convex algebras $A_{1}$ and $A_{2}$ and if $A_{3}$ is also a $Q$-algebra, then $M\left(A_{3}\right)$ is canonically homeomorphic to $M\left(A_{1}\right) \times M\left(A_{2}\right)$.

Proof. Theorems 1 and 2 and $\$ 1$, no. 2 of [4].

THEOREM 3. If $A_{1}$ and $A_{2}$ are complete commutative locally $m$-convex $Q$-algebras, then their complete projective tensor product is also such an algebra.

Proof. Let $A_{3}$ be the complete projective tensor product. In view of Theorem 2 and Remark 1, it suffices to show that $\sigma_{A_{3}}$ is continuous at zero. By Lemma 1 and Corollary 5.6 of [5], we have

$$
\begin{aligned}
\sigma_{A_{3}}(x) & =\sup _{m_{1}, m_{2}}\left|m_{1} \otimes m_{2}(x)\right| \\
& =\sup _{m_{2}} \sup _{m_{1}}\left|m_{1}\left(1 \otimes m_{2}(x)\right)\right| \\
& =\sup _{m_{2}} \sigma_{A_{1}}\left(1 \otimes m_{2}(x)\right)
\end{aligned}
$$


where 1 is the identity map on $A_{1}$ and we have written the tensor products of operators to denote its extension, which always exists by $\S 1$, no. 2 of [4]. Since $A_{1}$ is a $Q$-algebra, $\sigma_{A_{1}}$ is continuous at zero. There is a seminorm $\mu_{1}$, in the family defining the topology of $A_{1}$ such that $\sigma_{A_{1}}<\mu_{1}$. Thus

$$
\sigma_{A_{3}}(x) \leqq \sup _{m_{2}} \mu_{1}\left(1 \otimes m_{2}(x)\right) .
$$

Consider $x$ in $A_{1} \otimes A_{2}$, then

$$
x=\sum_{i=1}^{k} x_{1 i} \otimes x_{2 i}
$$

and

$$
\begin{aligned}
\sup _{m_{2}} \mu_{1}\left(1 \otimes m_{2}(x)\right) & \leqq \sup _{m_{2}} \sum_{i=1}^{k} \mu_{1}\left(x_{1 i}\right)\left|m_{2}\left(x_{2 i}\right)\right| \\
& \leqq \sum_{i=1}^{k} \mu_{1}\left(x_{1 i}\right) \sup _{m_{2}}\left|m_{2}\left(x_{2 i}\right)\right| \\
& \leqq \sum_{i=1}^{k} \mu_{1}\left(x_{1 i}\right) \sigma_{A_{2}}\left(x_{2 i}\right) .
\end{aligned}
$$

Again $A_{2}$ is a $Q$-algebra and so there is $\mu_{2}$ such that $\sigma_{A_{3}}<\mu_{2}$ and

$$
\sup _{m_{2}} \mu_{1}\left(1 \otimes m_{2}(x)\right) \leqq \sum_{i=1}^{k} \mu_{1}\left(x_{1 i}\right) \mu_{2}\left(x_{2 i}\right) .
$$

Varying the representation of $x$ and taking the infimum of the right side of this inequality, we obtain by Proposition 2 of [4] a seminorm, $\gamma$ of the family defining the projective topology on $A_{1} \otimes A_{2}$. For $x$ in the dense subset $A_{1} \otimes A_{2}$ we have

$$
\mu_{1}\left(1 \otimes m_{2}(x)\right) \leqq \gamma(x) .
$$

Since $\mu_{1}$ is continuous on $A_{1}$ while $1 \otimes m_{2}$ and $\gamma$ are continuous on $A_{3}$, this inequality holds for all $x$. Thus we have

$$
\sigma_{A_{3}}(x) \leqq \gamma(x)
$$

for $\gamma$ a seminorm in the family defining the topology of $A_{3}$, and $\sigma_{A_{3}}$ is continuous at zero.

Corollary 2. If $A_{1}$ and $A_{2}$ are complete commutative locally $m$ convex Q-algebras and $A_{3}$ is their complete projective tensor product, then $M\left(A_{3}\right)$ is canonically homeomorphic to $M\left(A_{1}\right) \times M\left(A_{2}\right)$.

Theorem 4. Let $A_{1}$ and $A_{2}$ be semisimple complete commutative 
locally m-convex Q-algebras, and $A_{3}$ their complete projective tensor product. $A_{3}$ is semisimple iff the extension to $A_{3}$ of $A_{1}^{\prime} \otimes A_{2}^{\prime}$ is dense in $A_{3}^{\prime}$.

Proof. By Remark 3, $A_{3}$ is semisimple iff the closed linear hull of $M\left(A_{3}\right)$ is $A_{3}^{\prime}$. Let $f$ be an element of $A_{3}^{\prime}$. If $A_{3}$ is semisimple there is, for every $\epsilon>0$ and every finite set $\left\{x_{i}\right\}$ of elements of $A_{3}$, a finite combination of elements of $M\left(A_{3}\right)$ approximating $f$ within $\epsilon$ on the set $\left\{x_{i}\right\}$. By Corollary 2, each element of $M\left(A_{3}\right)$ is in the extension to $A_{3}$ of $A_{1}^{\prime} \otimes A_{2}^{\prime}$ and this set is a linear space. Thus each neighborhood of $f$ in $A_{3}^{\prime}$ contains a member of $A_{1}^{\prime} \otimes A_{2}^{\prime}$.

Conversely, suppose $A_{3}^{\prime}$ is the closure of $A_{1}^{\prime} \otimes A_{2}^{\prime}$. For any $x \neq 0$ in $A_{3}$, there is $f$ in $A_{3}^{\prime}$ such that $f(x) \neq 0$. By hypothesis then, there are $f_{1}$ in $A_{1}^{\prime}$ and $f_{2}$ in $A_{2}^{\prime}$ such that $f_{1} \otimes f_{2}(x)=f_{1}\left(1 \otimes f_{2}(x)\right)=f_{2}\left(f_{1} \otimes 1(x)\right) \neq 0$. Since $A_{1}$ is semisimple, there is $m_{1}$ in $M\left(A_{1}\right)$ such that $m_{1}\left(1 \otimes f_{2}(x)\right)$ $=m_{1} \otimes f_{2}(x)=f_{2}\left(m_{1} \otimes 1(x)\right) \neq 0$. Since $A_{2}$ is semisimple, there is then $m_{2}$ in $M\left(A_{2}\right)$ such that $m_{2}\left(m_{1} \otimes 1(x)\right)=m_{1} \otimes m_{2}(x) \neq 0$. Thus $A_{3}$ is semisimple.

As an application of these theorems, one might consider $L_{D}^{1}(G)$ the completion of the algebra of functions from a locally compact Abelian group, $G$, to the indefinitely differentiable functions of compact support, $D$, with the usual topology, which are absolutely summable. (See $[4$, p. 58] and $[5$, p. 13].) Grothendieck (in $[4, \S 2$, no. 2]) has shown that as a space $L_{D}^{1}(G)$ is topologically isomorphic with the complete projective tensor product of $D$ and $L^{1}(G)$, the usual group algebra. (The extension to algebra isomorphism is easily carried out, as in [1].) From Theorem 3, we can conclude that $L_{D}^{1}(G)$ is a locally $m$-convex $Q$-algebra. Further the multiplicative functionals are homeomorphic to the direct product of the dual group of $G$ with the real line by Corollary 2 .

\section{REFERENCES}

1. B. Gelbaum, Tensor products of Banach algebras, Canad. J. Math. 11 (1959), $297-310$.

2. - Tensor products and related questions, Trans. Amer. Math. Soc. 103 (1962), 525-548.

3. J. Gil de Lamadrid, Uniform cross norms and tensor products of Banach algebras, Bull. Amer. Math. Soc. 69 (1963), 797-803.

4. A. Grothendieck, Produits tensoriels topologiques et espaces nucleaires, Mem. Amer. Math. Soc. No. 16 (1955), 331 pp.

5. E. A. Michael, Locally multiplicatively-convex topological algebras, Mem. Amer. Math. Soc. No. 11 (1952), 77 pp.

6. R. Schatten, $A$ theory of cross-spaces, Annals of Mathematics Studies No. 26, Princeton Univ. Press, Princeton, N. J., 1950. 
7. H. A. Smith, Tensor products of topological algebras, Dissertation, University of Pennsylvania, Philadelphia, Pa., 1964.

8. J. Tomiyama, Tensor products of commutative Banach algebras, Tôhoku Math. J. 12 (1960), 147-154.

9. T. Turumaru, On the direct-product of operator algebra. I, TOhoku Math. J. 4 (1953), 242-251.

University of Pennsylvania

\section{SQUARE ROOTS IN BANACH ALGEBRAS}

\section{TERRELL GARDNER}

A complex number which is not a nonpositive real number has a unique square root in the right half-plane. In this paper, we obtain an extension of this observation to general (complex) Banach algebras.

Since the elements we study are regular, and have logarithms, the existence of square roots is not at stake. Even the existence of roots having the desired spectral properties is evident. The uniqueness result we suppose to be new. It subsumes a somewhat weakened version of the classical result for positive, hermitian, bounded operators on a Hilbert space (that such an operator has a unique positive hermitian and bounded square root): our theorem would apply only to the positive definite (regular) case.

Whether the results of this paper can be extended to the case of not necessarily regular elements of a Banach algebra, we do not at present know.

Definition. A subset $A$ of the complex field will be called positive if $x \in A$ implies $x>0 ; A$ is weakly positive if $x \in A$ implies $\Re(x)>0$. Let $\Sigma$ denote the complement, in the complex plane, of the nonpositive real numbers.

Theorem. Let $\mathfrak{B}$ be a Banach algebra, and $b$ an element of $\mathfrak{B}$ with spectrum $\mathrm{Sp}(b)$ contained in $\Sigma$. Then there exists in $\mathfrak{B}$ a unique square root of $b$ with weakly positive spectrum. If $\mathrm{Sp}(b)$ is real (hence positive), so is $\mathrm{Sp}\left(b^{1 / 2}\right)$.

Proof. Existence: We assume first that $\mathfrak{B}$ has an identity ele-

Received by the editors May 24, 1965. 